Research Paper

\title{
miR-26b enhances the sensitivity of hepatocellular carcinoma to Doxorubicin via USP9X-dependent degradation of p53 and regulation of autophagy
}

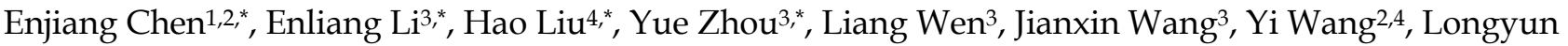 \\ $\mathrm{Ye}^{5}$, Tingbo Liang, $2,3,6,7 \bowtie$ \\ 1. The Second Affiliated Hospital of Zhejiang University, School of Medicine, Hangzhou, China. \\ 2. Zhejiang Provincial Key Laboratory of Pancreatic Disease, Hangzhou, China. \\ 3. Department of Hepatobiliary and Pancreatic Surgery, The First Affiliated Hospital, Zhejiang University School of Medicine, Hangzhou, China. \\ 4. Department of Medical Oncology, Tongde hospital of Zhejiang Province, Hangzhou, Zhejiang, 310012, China. \\ 5. Department of Pancreatic Surgery, Fudan University Shanghai Cancer Center, Shanghai 200032, China. \\ 6. Zhejiang Provincial Innovation Center for the Study of Pancreatic Disease, Hangzhou, China. \\ 7. Zhejiang Clinical Research Center of Hepatobiliary and Pancreatic Diseases, Hangzhou, China. \\ * These authors contributed equally to this work. \\ $\triangle$ Corresponding author: Tingbo Liang, Department of Hepatobiliary and Pancreatic Surgery, The First Affiliated Hospital, Zhejiang University School of \\ Medicine, Hangzhou, 310003, China. Email: liangtingbo@zju.edu.cn \\ (c) The author(s). This is an open access article distributed under the terms of the Creative Commons Attribution License (https://creativecommons.org/licenses/by/4.0/). \\ See http://ivyspring.com/terms for full terms and conditions.
}

Received: 2020.08.27; Accepted: 2021.01.16; Published: 2021.02.08

\begin{abstract}
Multi-drug resistance is a major challenge to hepatocellular carcinoma $(\mathrm{HCC})$ treatment, and the over-expression or deletion of microRNA (miRNA) expression is closely related to the drug-resistant properties of various cell lines. However, the underlying molecular mechanisms remain unclear. CCK-8, EdU, flow cytometry, and transmission electron microscopy were performed to determine cell viability, proliferation, apoptosis, autophagic flow, and nanoparticle characterization, respectively. In this study, the results showed that the expression of miR-26b was downregulated following doxorubicin treatment in human HCC tissues. An miR-26b mimic enhanced HCC cell doxorubicin sensitivity, except in the absence of $\mathrm{p} 53$ in Hep3B cells. Delivery of the proteasome inhibitor, MG132, reversed the inhibitory effect of miR-26b on the level of p53 following doxorubicin treatment. Tenovin-1 (an MDM2 inhibitor) protected p53 from ubiquitination-mediated degradation only in HepG2 cells with wild type p53. Tenovin-1 pretreatment enhanced HCC cell resistance to doxorubicin when transfected with an miR-26b mimic. Moreover, the miR-26b mimic inhibited doxorubicin-induced autophagy and the autophagy inducer, rapamycin, eliminated the differences in the drug sensitivity effect of miR-26b. In vivo, treatment with sp94dr/miR-26b mimic nanoparticles plus doxorubicin inhibited tumor growth. Our current data indicate that miR-26b enhances HCC cell sensitivity to doxorubicin through diminishing USP9X-mediated p53 de-ubiquitination caused by DNA damaging drugs and autophagy regulation. This miRNA-mediated pathway that modulates HCC will help develop novel therapeutic strategies.
\end{abstract}

Key words: microRNA-26b; hepatocellular carcinoma; Doxorubicin; USP9X; p53.

\section{Introduction}

Hepatocellular carcinoma (HCC) has a high-incidence and aggressive types of cancer, with increasing global incidence, ranking second in mortality worldwide [1, 2]; however, several treatment methods can cure patients suffering from HCC, including liver transplantation, radiotherapy, surgery, and interventional therapy. In addition, chemotherapy is often used before or after the above-mentioned treatment strategies. Due to the ineffective chemotherapy or multidrug resistance (MDR) procedures, patients with advanced HCC have a poor prognosis or clinical outcomes [3]. Therefore, it is necessary to identify a new molecular mechanism 
underlying the drug resistance and develop effective treatment strategies for HCC.

MicroRNAs (miRNAs) are a type of evolutionarily conserved non-coding small RNA with the function of regulating gene expression at the stage of translation, and has been considered to be abnormally expressed in several human cancers $[4,5]$. Accumulating research indicates that some miRNAs display aberrant expression in various cancer cells (e.g., HCC, breast cancer, and human non-small lung cancer [NSCLC]) [6-8]. In several cancers, miRNAs act as tumor suppressors or oncogenes related to the multiple pathways and cell functionality involved in development and progression of cancer (e.g., cell cycle, proliferation, and apoptosis) $[9,10]$. It has been demonstrated that miRNA-26b (miR-26b) is involved in a variety of malignant cancer progressions as tumor oncogenes or suppressors (e.g., lung, HCC, melanoma, ovarian, and colorectal cancer) [11-15]. Importantly, the aberrant expression of miR-26b is significantly associated with cell proliferation, metabolism, and apoptosis [16]. These results are largely due to their effect of the activation of multiple related downstream target genes on angiogenesis, the process of cell-cycle development and anti-apoptosis $[17,18]$.

Ubiquitination has been found to be a key regulatory mechanism in multiple biological processes and controls almost all aspects of protein function through the reversible post-translational modification of cellular proteins by the activity of ubiquitylating and deubiquitylating enzymes (DUBs)[19, 20]. Ubiquitin-specific proteases (USPs) represent the largest DUB group of ubiquitinating enzymes, which function to specifically depolymerize ubiquitin from ubiquitinated substrates and play an important role in the ubiquitin system. Ubiquitin-specific protease-9 (USP9X) acts as a member of the USP family to regulate cell sensitivity to chemo and radiotherapies. Moreover, USP9X was found to be overexpressed in different types of lymphoma, such as diffuse large B-cell and follicular lymphoma, as well as multiple myeloma [21]. WP1130 acts as a partially selective DUB inhibitor, and is considered to be a potential chemosensitizer due to its inhibitory activity on USP9X de-ubiquitination [22, 23]. In addition, WP1130 can promote the down-regulation of myeloid cell leukemia 1 protein (Mcl-1) and increase the sensitivity of tumor cells to chemotherapy [24]. WP1130 also increases sensitivity to doxorubicin via degrading p53 by a USP9X-dependent mechanism in HCC cells [22]. Autophagy is a highly complex process, which plays a dual role in the processes of tumor treatment, regulation of cell survival, differentiation, apoptosis, and a series of other biological processes [25, 26]. Moreover, miRNA may participate in the resistance of various cancers to therapy $[27,28]$. It has been previously reported that $\mathrm{miR}-26 \mathrm{a} / \mathrm{b}$ enhances apoptosis and sensitivity in HCC via inhibiting ULK1 [29]. Xinming et al. demonstrated that the overexpression of miR-30 enhances cisplatin sensitivity through the inhibition of autophagy [30].

This study evaluated the miR-26b-induced sensitivity of HCC cells to doxorubicin. Furthermore, we confirmed the specific mechanism of miR-26b on HCC cells through USP9X down-regulates p53 upregulation caused by DNA damaging drugs and the regulation of autophagy.

\section{Materials and Methods}

\section{Cell culture and the Source of HCC tissue}

Human HCC cells (HepG2 (Wild-type p53), Hep3B (p53 deletion) SNU387 and SNU449 (p53 mutation) were supplemented from ATCC. All of the HCC cells were maintained in different medium containing $\quad 10 \% \quad$ FBS(GIBCO) and $1 \%$ penicillin/streptomycin (Sigma), such as SNU449 and SNU387 (RPMI1640 medium), HepG2 (DMEM), Hep3B (MEM). The cells were cultured in a humidified environment containing $5 \% \mathrm{CO} 2$ at $37^{\circ} \mathrm{C}$. The adjacent tissues and HCC tissues were obtained from The Second Affiliated Hospital of Zhejiang University, School of Medicine.

\section{Transient transfection with an miR-26b mimic and inhibitor}

According to the manufacturer's instructions, Lipofectamine 2000 (Invitrogen,) was used to transfect the HCC cells. For the SP-94dr/miR-26b nanoparticle infection, miR-26b mimics and SP-94dr were mixed and allowed to rest for $15 \mathrm{~min}$, after which they were added to the plates. RiboBio (Ribobo Co., Ltd.) and Santa Cruz Biotechnology (Santa Cruz, CA, USA) provided the related transient transfection products (miR-26b mimic, miR-26b inhibitor, and negative control: RiboBio; USP9X siRNA, negative siRNA : Santa Cruz).

\section{Cell viability assay}

Cell Counting Kit-8 (CCK-8; Dojindo) was used to determine the related cell viability according to product description. Briefly, HCC cells $(3 \times 103$ cells/well) were seeded into 96-well plates and cultured for $24 \mathrm{~h}$. The culture medium was replaced by $10 \%$ FBS-medium containing the indicated doxorubicin concentration. After a further incubation for $48 \mathrm{~h}, 10 \mu \mathrm{L}$ of CCK-8 solution was added, the cells were incubated for an additional $3 \mathrm{~h}$, and then absorbance at $450 \mathrm{~nm}$ was measured using an MRX II 
microplate reader (Dynex Technologies, Chantilly, USA). Relative cell viability was calculated as a percentage of the untreated controls.

\section{Western Blot analysis}

The HCC cells transfected with the miR-26b mimic, or USP9X siRNA were lysed with RIPA lysis buffer (Beyotime, Shanghai, China), centrifuged at $12,000 \mathrm{rpm}$ for $10 \mathrm{~min}$ at $4^{\circ} \mathrm{C}$, and the supernatants were collected. After collecting the protein samples, the protein concentration of each sample was determined to ensure that the amount of each protein sample was consistent. Then protein was quantified using a BCA protein kit. A 10\% SDS-PAGE gel was prepared and the same amount of protein was loaded in to each well. The separated proteins were subsequently transferred onto a PVDF membrane. The membrane was slowly agitated on a shaker, blocked in 5\% TBST at room temperature for $60 \mathrm{~min}$, washed three times, and incubated with a 1:1,000 diluted primary antibody (anti-USP9X, anti-p53; Abcam) in TBST overnight at $4^{\circ} \mathrm{C}$ on a side-swing shaker. The membrane was washed three times for 5 10 min with slow shaking on the side-swing shaker before incubating with the corresponding secondary antibody (Abcam; 1:2000) at room temperature or $4^{\circ} \mathrm{C}$ with slow shaking for $1 \mathrm{~h}$ on a side swinging shaker. Finally, the detection of the proteins was performed using an ECL color kit. A gray value quantification analysis of the protein bands was performed using ImageJ software (National Institutes of Health, Bethesda, MD, USA).

\section{Quantitative real-time reverse transcription-PCR (RT-PCR)}

Total RNA was extracted using TRIzol reagent (Invitrogen) according to the manufacturer's instructions. Reverse transcription was performed to obtain the first strand of cDNA using a PrimeScript ${ }^{\circledR}$ $\mathrm{RT}$ reagent kit (Takara). The relative level of miR-26b was normalized to U6. All qRT-PCR reactions were performed using SYBR Green Master Mixes (Thermo Fisher). The results were analyzed using the $2^{-\Delta \Delta C t}$ method. The primer sequences are listed as follows: p53: Forward 5'-TCAGCATCTTATCCGAGTGG AA-3'; Reverse 5'-TGTAGTGGATGGTGGTACAG TCA-3'. USP9X: Forward 5'-CAATGGATAGATCGC TTTATA-3'; Reverse 5'-CTTCTTGCCATGGCCTTA AAT-3'. hsa-miR-26b mimics: Forward 5'-UUCAAG UAAUUCAGGAUAGGU-3'; Reverse 5'-CUAUCC UGAAUUACUUGAAUU -3'. hsa-miR-26b inhibitor: 5'-ACCUAUCCUGAAUUACUUGAA-3'.

\section{Cell proliferation analysis}

Proliferation of HCC cells was determined using an EdU staining proliferation kit according to manufacturer's (Abcam) protocols. The HCC cells $(3 \times$ 103 cells/well) transfected with or without miR-26b mimics were seeded into 96-well plates and cultured for $24 \mathrm{~h}$. Later, the cells were treated with or without doxorubicin for $24 \mathrm{~h}$ and incubated with $20 \mu \mathrm{M}$ EdU for $3 \mathrm{~h}$. DNA (blue) was stained with Hoechst 33342. Green cells indicated EdU/Hoechst-positive cells.

\section{Flow cytometry}

The apoptosis rate of the HCC cells was determined using an Annexin V-FITC Apoptosis Detection Kit (Abcam). Briefly, HCC cells (treated as above) were harvested by trypsinization, rinsed with ice-cold PBS, and centrifuged to remove the supernatant. The cells were resuspended in $100 \mu \mathrm{L} 1 \times$ binding buffer and incubated with Annexin V-FITC for $15 \mathrm{~min}$ in the dark at room temperature. Finally, flow cytometry was used to determine the number of apoptotic cells based on the B2 and B4 quadrants.

\section{Transmission electron microscopy}

Autophagosome formation of was examined using transmission electron microscopy (TEM). The harvested cells were fixed with $2.5 \%$ glutaraldehyde at $4^{\circ} \mathrm{C}$ overnight, and post-fixed in $1 \%$ buffered osmium tetroxide for $2 \mathrm{~h}$. After dehydration in series of ethanol, the samples were embedded in an epoxy resin, followed by staining with uranyl acetate and lead citrate. The stained sections were examined under a transmission electron microscope (H800; Hitachi).

\section{Luciferase Reporter Assay}

The amplified of the wild type USP9X-3'UTR or mutant USP9X-3'UTR fragment was cloned into the pGL3 vector containing the firefly luciferase reporter gene (Promega, Madison, WI, USA). For the luciferase reporter assays, HEK293 cells were plated in 96-well plates and transiently co-transfected with $200 \mathrm{ng}$ firefly luciferase construct, 4 ng pRL-TK Renilla luciferase plasmid, and $50 \mathrm{nM}$ miR-26b-mimic according to the manufacturer's protocol supplied with a luciferase reporter assay kit (Promega Madison, WI). After $48 \mathrm{~h}$ transfection, the relative Renilla luciferase activity (firefly luciferase/Renilla luciferase) was measured with a dual luciferase reporter assay (Promega, USA).

\section{Nanoparticle characterization}

SP-94dr/miR-26b nanoparticles were analyzed for hydrodynamic diameters and zeta-potential using a laser particle analyzer at $25^{\circ} \mathrm{C}$. TEM was performed at an accelerating voltage of $80 \mathrm{kV}$ with a Philips TECNAL-10 (Eindhoven). 


\section{MiRNA stability test}

A total of $20 \mu \mathrm{M}$ miR-26b and SP-94dr (SP-94dr: miR-26b) was mixed at a molar ratio of 20:1 and incubated at room temperature for $10 \mathrm{~min}, 1 \mu \mathrm{L}$ RNase A (diluted to $0.001 \mu \mathrm{g} / \mu \mathrm{L}$, Takara) was added and incubated for the indicated times at room temperature and separated on a $2 \%$ agarose gel.

\section{Nude mouse xenograft model}

BALB/c nu/nu mice (4-5 weeks old) were obtained from GemPharmatech Co., Ltd (Nanjing, China). Patient-derived tumor xenografts (PDTX) were used to establish the tumor model. PDTX maintains the morphological, structural, and molecular characteristics of the tumor cells. Furthermore, PDTX uses a transplant method that is closer to the tumor microenvironment. In our research group, the rate of successful PDTX tumor inoculation was about $10 \%, 20$ case HCC tumor tissue can be successfully modeled in 2 cases. Tumor fragments of 1 $\mathrm{mm} 3$ were cut from resected tumors and inoculated into the mice. After the tumor had formed, the tumor tissue was obtained, sliced, and repeatedly inoculated into $32 \mathrm{BALB} / \mathrm{c}$ nude mice, at last we used the tumor into BALA/c nude mice to do the further research. When the diameter of tumors reached a size of $0.5 \mathrm{~cm}$, and the tumor volume reached about $50 \mathrm{~mm}^{3}-100$ $\mathrm{mm}^{3}$, the mice were randomly divided into four groups: 1) Control (saline); 2) Doxorubicin (2 mg/kg); 3) miR-26b mimic (sp-94dr) (miR-26b mimic: $10 \mu \mathrm{g}$, sp-94dr: $200 \mu \mathrm{g}$, the injection dose of the mixture was $100 \mu \mathrm{L}$ each); and 4) miR-26b mimic (sp-94dr) + Doxorubicin ( $\mathrm{n}=6$ per group). In this experiment, the peptide nanoparticles formed by the mixture of mir-26b mimics and sp-94dr peptides were injected into the tail vein. The control (normal saline), Doxorubicin, miR-26b mimic, or miR-26b mimic plus Doxorubicin groups were administered multi-point intratumoral injections every other day for two weeks. The tumor volume was recorded every two days (Tumor volume $\left(\mathrm{mm}^{3}\right)=$ length $\times$ width $\left.^{2} / 2\right)$. The mice were sacrificed on Day 15 after treatment and the tumors were weighed.

\section{Immunocytochemistry}

Immunohistochemical staining was performed to determine the level of $\mathrm{Ki}-67$ expression. The paraffin sections were deparaffinized in water, blocked with $5 \%-10 \%$ normal goat serum (diluted in PBS) for $10 \mathrm{~min}$ at room temperature, and incubated with an anti-Ki-67 antibody (1:500, Abcam) at $4^{\circ} \mathrm{C}$ overnight. The samples were washed with PBS three times, incubated with horseradish peroxidase (HRP) for $30 \mathrm{~min}$ at $37^{\circ} \mathrm{C}$, washed again, and diaminobenzidine (DAB) was added and developed for $10 \mathrm{~min}$. Finally, hematoxylin was used as a counterstain, the sections were dehydrated, sealed in film, and observed under light microscopy (Olympus).

\section{TUNEL analysis}

Apoptosis in paraffin-embedded mouse tissue sections (5-mm) was determined using an in situ cell death detection kit (Roche). Briefly, the cells were fixed in $4 \%$ paraformaldehyde for $24 \mathrm{~h}$. After conventional dehydration, wax infiltration, and paraffin embedding, the cells were subsequently incubated with a mixture of a fluorescent labeling solution of $450 \mu \mathrm{L}$ dUTP and $50 \mu \mathrm{L}$ TdT enzyme at $37^{\circ} \mathrm{C}$ for $1 \mathrm{~h}$. Next, $50 \mu \mathrm{L}$ converter-POD was added to the specimen, a cover glass or parafilm was added and reacted in a dark humidified box at $37^{\circ} \mathrm{C}$ for 30 min. The samples were subsequently treated with diaminobenzidine for $10 \mathrm{~min}$ at $15^{\circ} \mathrm{C}-25^{\circ} \mathrm{C}$, counterstained with hematoxylin (to identify the cell nuclei), dehydrated in a gradient series, vitrified with dimethylbenzene, and finally, mounted with neutral balsam. The prepared samples were evaluated under an optical microscope.

\section{Statistical analysis}

The experimental data are presented as the mean \pm SD. Comparisons between two groups or multiple groups were analyzed using a two-tailed Student's $t$-test and a one-way analysis of variance (ANOVA) followed by Tukey's post hoc test, respectively. A value of $\mathrm{P}$ less than 0.05 was identified as a statistically significant difference. Statistical analysis was performed using GraphPad Prism 8. All of the experiments were repeated at least three times.

\section{Results}

\section{Treatment with miR-26b enhances HCC cell sensitivity to doxorubicin}

QRT-PCR was used to analyze changes in miRNA before and after doxorubicin treatment in SNU449 and SNU387 cells. MiR-26b expression was the lowest in the cells treated with doxorubicin (Figure 1A). To reveal the role of miR-26b on HCC cell doxorubicin sensitivity, qRT-PCR was used to determine the level of miR-26b in HCC cells, and we found that the level of miR-26b was down-regulated after treatment with doxorubicin (Figure 1B). Treatment with the miR-26b mimic could enhance the sensitivity of SNU449, HepG2, and SNU387 cells to doxorubicin, whereas the miR-26b mimic had no effect on the sensitivity to doxorubicin in Hep3B cells (Figure 1C). 
A

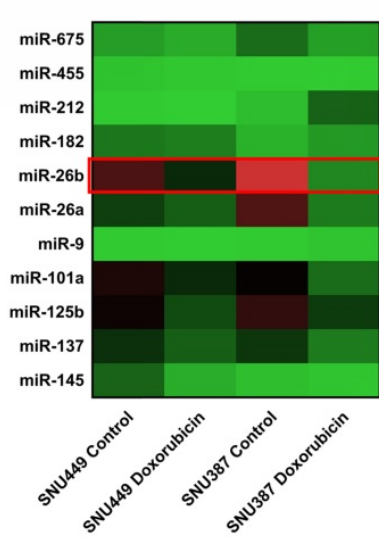

B

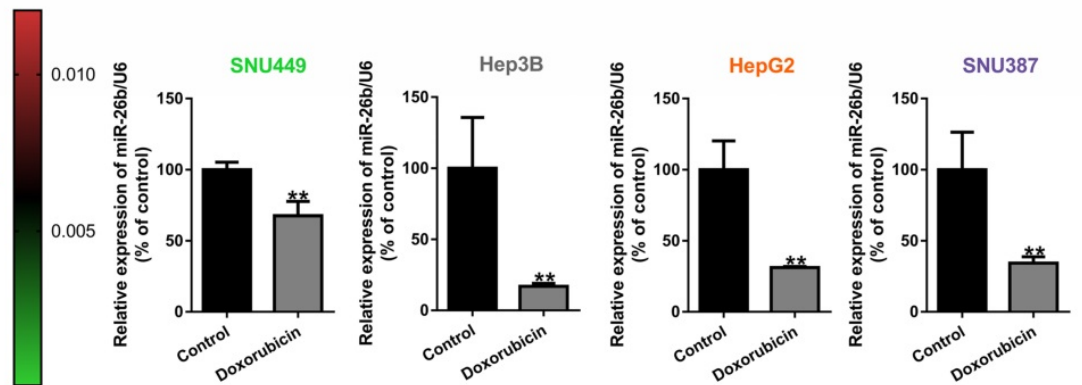

C
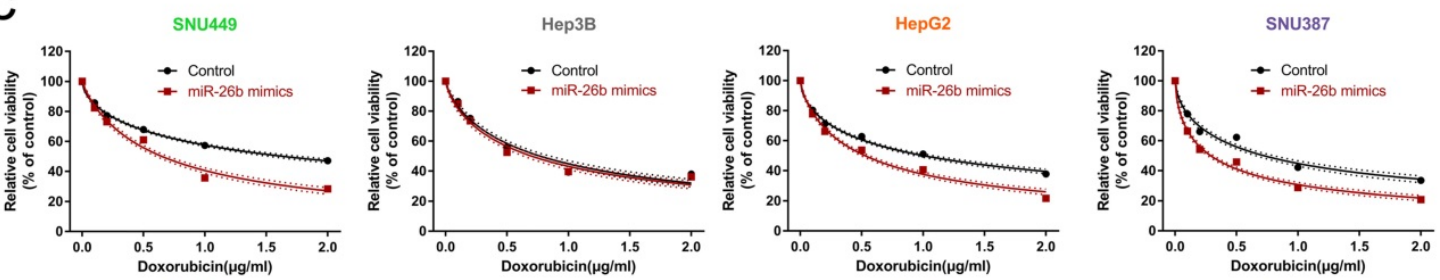

D
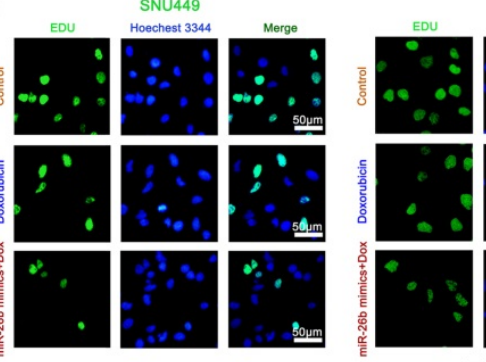

Нерзв

E
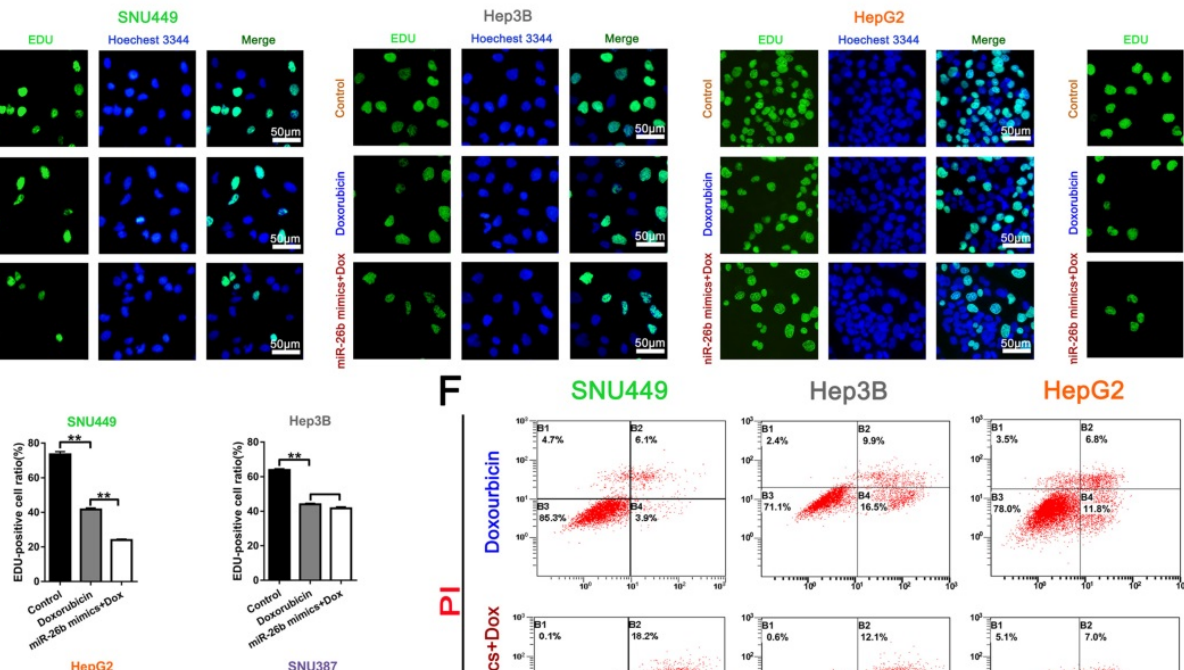

SNU387

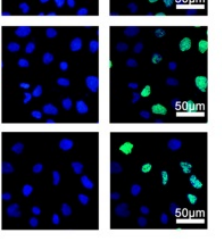

F

SNU449
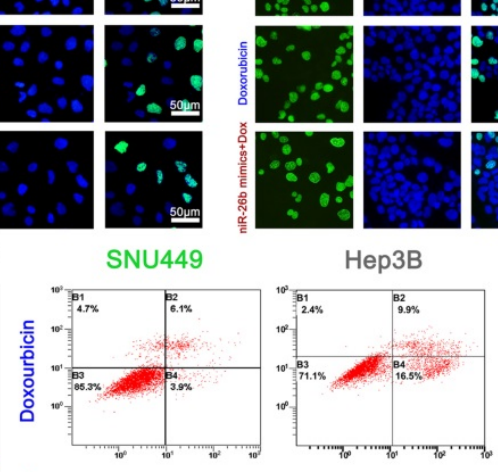

HepG2
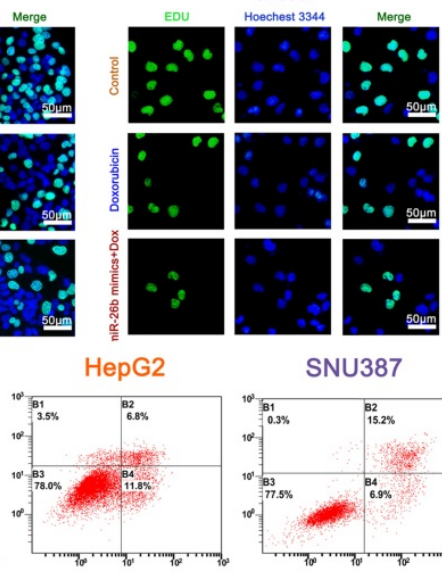

SNU387

$\bar{\alpha}$
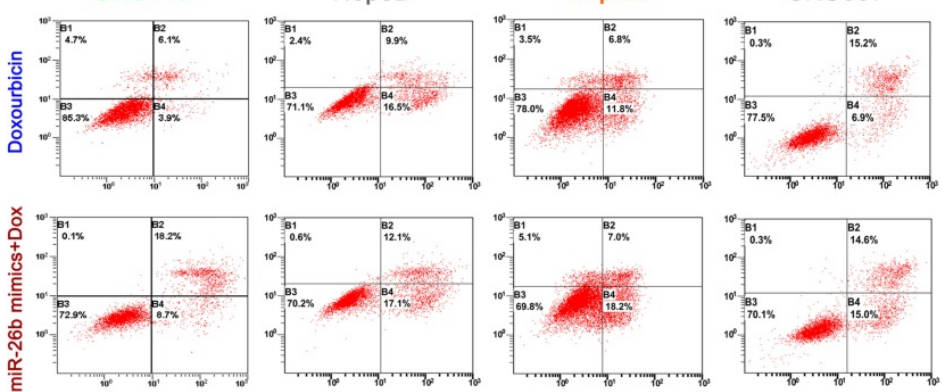

Hep
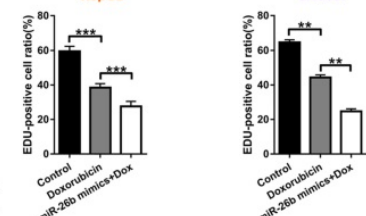

G
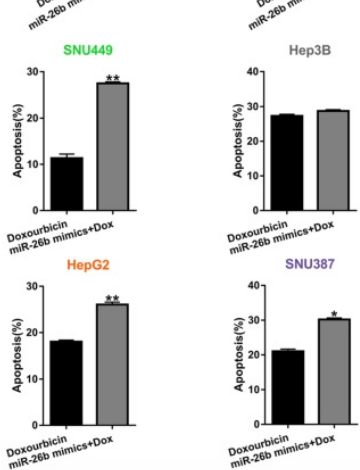

$\mathrm{H}$

Annexin V

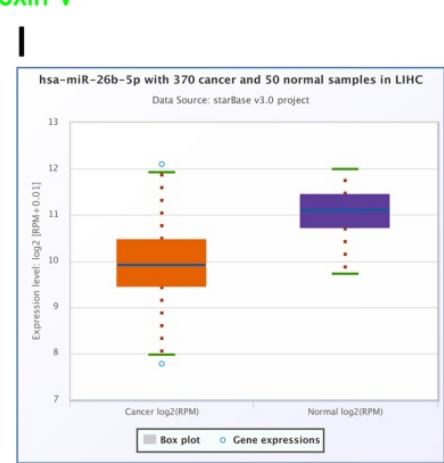

Figure 1. miR-26b enhances HCC cell sensitivity to doxorubicin. A. QRT-PCR analysis of the changes in miRNA after treatment with doxorubicin in SNU449 and SNU387 cells. B. The level of miR-26b was determined following treatment with or without Doxorubicin by qRT-PCR in HCC cells. **P < 0.01 . C. A CCK-8 assay analysis showed that treatment with an miR-26b mimic can enhance the sensitivity of HCC cells to doxorubicin, with the exception of Hep3B cells. D and E. An EdU incorporation assay of cellular proliferation in different treatment groups. **P $<0.01$. F and $\mathbf{G}$. The apoptosis ratio was determined by flow cytometry. *P $<0.05$; **P $<0.01$. $\mathbf{H}$. QRT-PCR used to determine miR-26b expression in adjacent cancer and adjacent tissues. I. We used StarBase $v 3.0$ project to analyze the level of miR-26b in normal and cancer tissues. 
The IC50 values are listed in Table 1. EdU incorporation assay and the flow cytometry analysis indicated that the miR-26b mimic combined with doxorubicin could reduce the cellular proliferation rate and increase the number of apoptotic cells in HCC cells, except Hep3B cells (Figure 1D-G). Almost immediately, we found that miR-26b was expressed to a greater extent in the adjacent tissues (Figure 1H). StarBase v.3 was used to analyze miR-26b expression in liver hepatocellular carcinoma (LIHC) [31, 32], showing that miR-26b was expressed to a greater extent in the normal tissues compared to that in cancer tissues (Figure 1I).

Table 1. IC 50 values for doxorubicin in HCC cell lines with or without miR-26b mimic siRNA treatment.

\begin{tabular}{lll}
\hline HCC cell line & $\mathrm{IC}_{50}(\mu \mathrm{g} / \mathrm{mL}) \boldsymbol{\Lambda}$ & \\
\cline { 2 - 3 } & Doxorubicin & Doxorubicin + miR-26b mimic \\
\hline SNU449 & $1.644 \pm 0.06229$ & $0.6510 \pm 0.02349^{*}$ \\
Hep3B & $0.7701 \pm 0.03647$ & $0.7012 \pm 0.03443$ \\
HepG2 & $1.007 \pm 0.03633$ & $0.5273 \pm 0.01735^{*}$ \\
SNU387 & $0.7263 \pm 0.03554$ & $0.2903 \pm 0.01201^{*}$
\end{tabular}

$\triangle \mathrm{IC}_{50}$ concentrations of doxorubicin $(\mu \mathrm{g} / \mathrm{mL} ;$ mean $[95 \% \mathrm{CI}]) .{ }^{*} \mathrm{P}<0.05 \mathrm{vs}$. Doxorubicin.
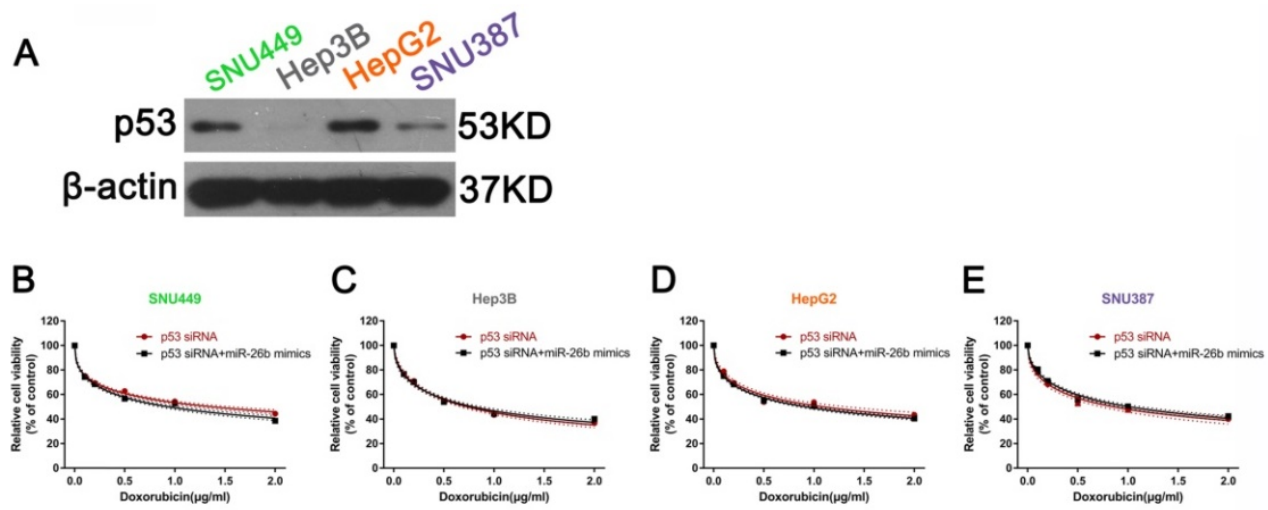

$\mathbf{F}$
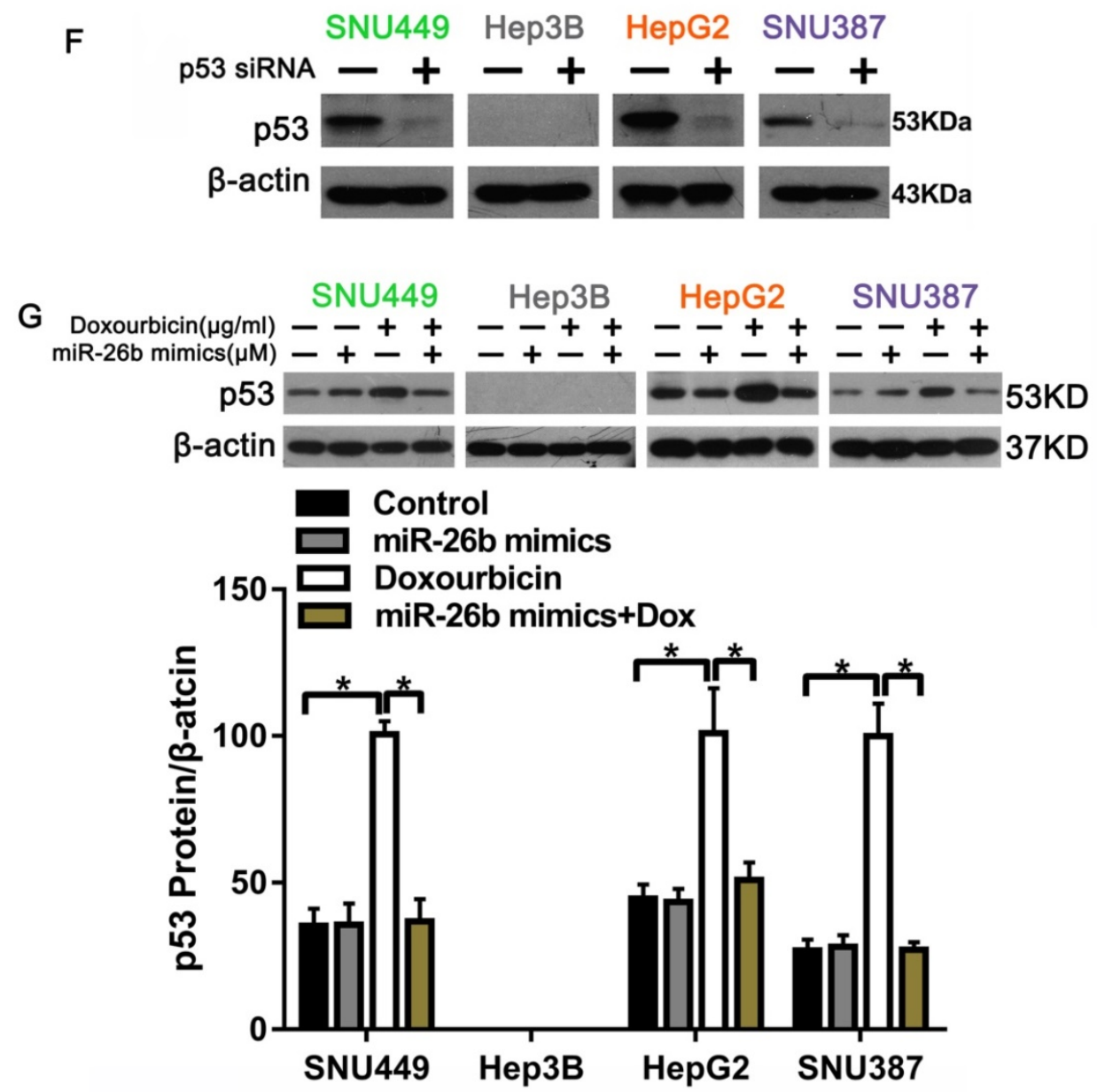

Figure 2. p53 was related to the sensitivity of HCC cells to doxorubicin. A. P53 protein expression was detected by Western blot. B - E. The cell viability was examined in doxorubicin, or miR-26b mimic plus doxorubicin treated cells following transfection with p53 siRNA. 


\section{The relationship between p53 and doxorubicin sensitivity in HCC cells}

Our previous research demonstrated that WP1130 increases doxorubicin sensitivity in HCC cells through USP9X-dependent p53 degradation [22]. We assumed that whether or not miR-26b had an effect on doxorubicin treatment was due to p53 degradation. To test this theory, we first examined p53 expression in different HCC cells by Western blot. The results showed that the HCC cells expressed p53, except Hep3B cells (Figure 2A). We also transfected p53 siRNA into HCC cells and observed the level of cell viability between doxorubicin treatment alone and the miR-26b mimic plus doxorubicin treatment, revealing that the effect of miR-26b mimic enhanced doxorubicin sensitivity in HCC cells was inhibited following transfection with p53 siRNA (Figure 2B-E). The Western blot confirmed the detection interference efficiency of p53 (Figure 2F). As shown in Figure 2G, as compared to doxorubicin treatment alone, p53 expression was down-regulated after transfection with the miR-26b mimic and interaction with doxorubicin.

\section{miR-26b enhances HCC cell sensitivity to doxorubicin via USP9X-dependent p53 degradation}

We next further explored the mechanism by which miR-26b regulates doxorubicin-induced p53 expression. As shown as in Figure 3A, p53 was down-regulated in the miR-26b mimic + doxorubicin group, whereas p53 was re-covered following treatment with MG132 + miR-26b MIMIC + doxorubicin group, suggesting that MG132 could block the activity of miR-26b, and miR-26b may play a role in promoting p53 ubiquitination degradation. In addition, the CCK-8 analysis showed that in all of the HCC cells, except in the HepG2 cells treated with Tenovin-1 (a wild type p53 activator) for $2 \mathrm{~h}$, miR-26b could reduce the sensitivity to doxorubicin in HepG2 cells (Figure 3B). We further explored the mechanism by which miR-26b regulates doxorubicin-induced p53. Targetscan software predicted that miR-26b could bind to the USP9X promoter region and dual luciferase reporter gene experiments verified that miR-26b overexpression decreased the luciferase activity of the constructs containing the wild type USP9X 3'UTRs, Mut (1132-1139), or (1317-1323) USP9X 3'UTRs, whereas Mut(both) USP9X 3'UTRs remained unchanged (Figure $3 \mathrm{C}$ ). We assumed that miR-26b could regulate p53 degradation by USP9X. A Western blot revealed that USP9X expression was down-regulated after transfection with the miR-26b mimic (Figure 3D). The level of p53 expression between the doxorubicin + USP9X siRNA group and doxorubicin + USP9X siRNA + miR-26b mimic were not significantly different (Figure 3E). Furthermore, the interference efficiency of USP9X was determined by Western Blot (Figure 3F).

\section{miR-26b inhibits doxorubicin-induced autophagy}

During autophagy, the level of LC3II protein expression serves as a read-out of the number of autophagosomes, and the conversion of LC3I to LC3II (LC3II/LC3I) is indicative of autophagic activity [33]. The autophagy receptor, sequestosome 1 (SQSTM1, p62), connected the autophagic cargo to the autophagic membrane, and can be used as another widely marker of autophagic flux [34]. The Western blot analysis showed that the combination of doxorubicin and miR-26b mimics could down-regulate the increased expression of USP9X, p53, and LC3II/LC3I following doxorubicin treatment, and up-regulated the decrease in p62 expression induced by doxorubicin (Figure 4A). The level of autophagy was determined by the total number of autophagosomes (mRFP + GFP) and autolysosomes (mRFP). As shown in Figure 4B and C, treatment with miR-26b mimic + doxorubicin could reduce the increase of doxorubicin-induced the total number of autophagosomes and autolysosomes in HepG2 and SNU449 cells (Figure 4B-D). The characteristic autophagic ultrastructures were determined by TEM analysis. As shown in Figure 4E and F, compared with the control group, the red color indicated that the number of autophagosome was increased following doxorubicin treatment, whereas miR-26b over-expression could reduce the increase in the number of autophagosome induced by doxorubicin. Furthermore, the miR-26b mimic could inhibit autophagy activity. CCK-8 detected differences in the sensitivity between different groups (transfected with or without miR-26b mimics, and miR-26b mimics combined with rapamycin) to doxorubicin, showing that treatment with the miR-26b mimics enhanced the chemosensitivity of the HCC cells. This effect disappeared after the addition of rapamycin (Figure 4G).

\section{Sp94dr/miR-26b mimic nanoparticles enhanced HCC cell doxorubicin sensitivity in vivo}

We conducted further in vivo experiments using liver cancer-targeting peptides, sp94dr and miR-26b self-assembled nanoparticles to conduct experiments due to the cumbersome nature of drug administration in vivo. Treatment with the sp94dr/miR-26b mimic nanoparticles plus doxorubicin had an inhibitory 
effect on tumorigenesis in vivo, similar to the in vitro studies (Figure 5A, C and D). There was no significant change in the body weight of the mice after treatment in the different groups (Figure 5B). Ki-67 staining and a TUNEL assay found that treatment with the sp94dr/miR-26b mimic nanoparticles plus doxorubicin significantly decreased the tumor cell proliferation rates and increase tumor cell apoptosis compared to the other groups (Figure 5E-F).

A
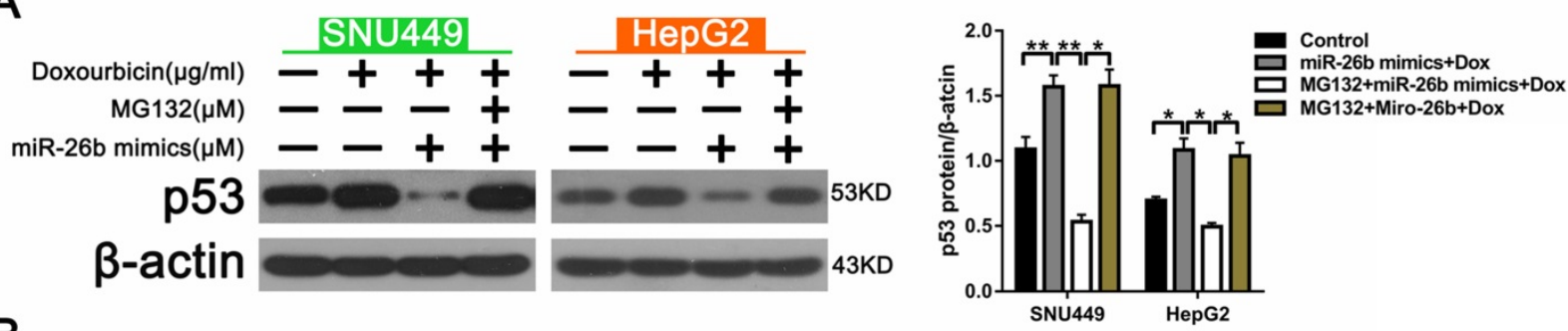

B

SNU449

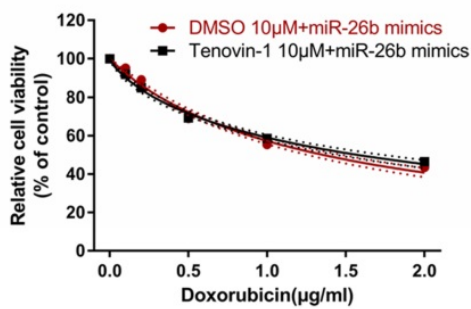

C

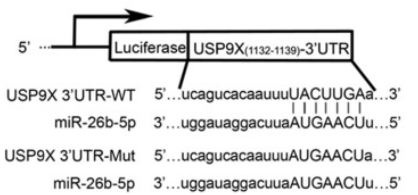

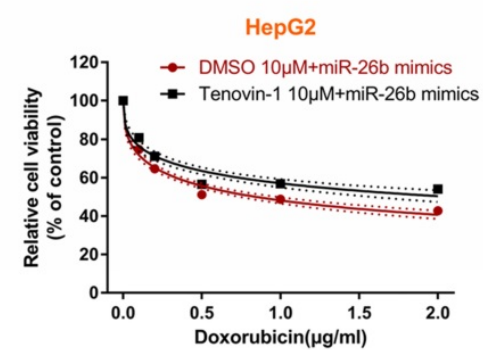
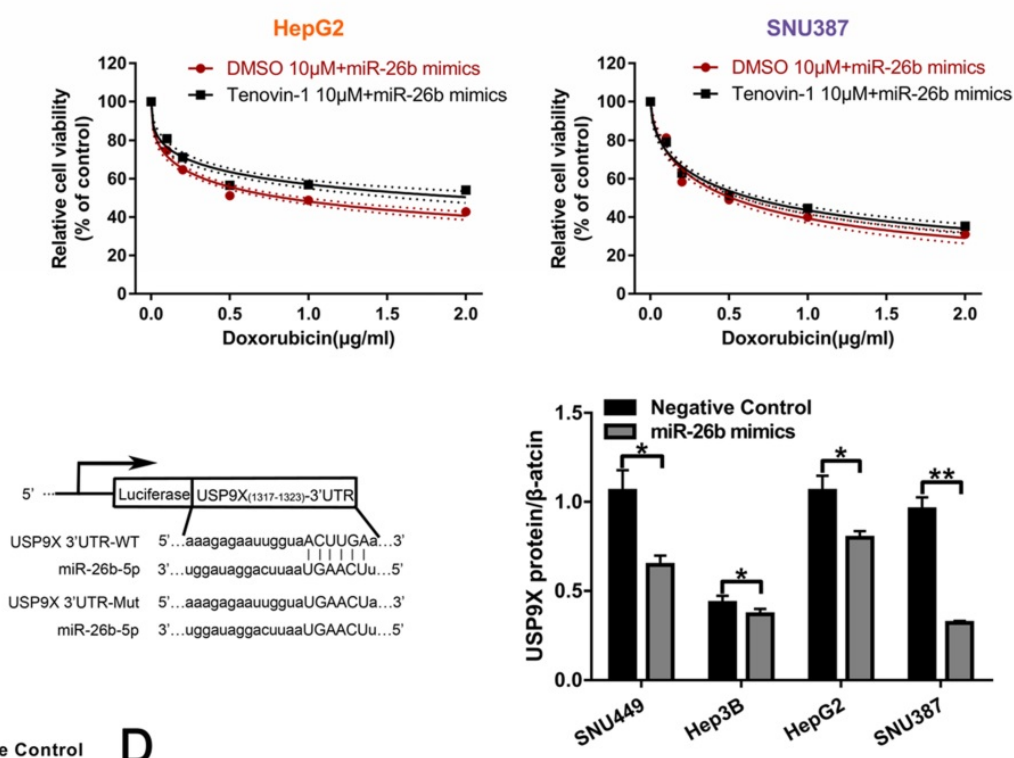

miR-26b-5p $\quad 3^{\prime} \ldots$...uggauaggacuuaaUGAACUu...5'

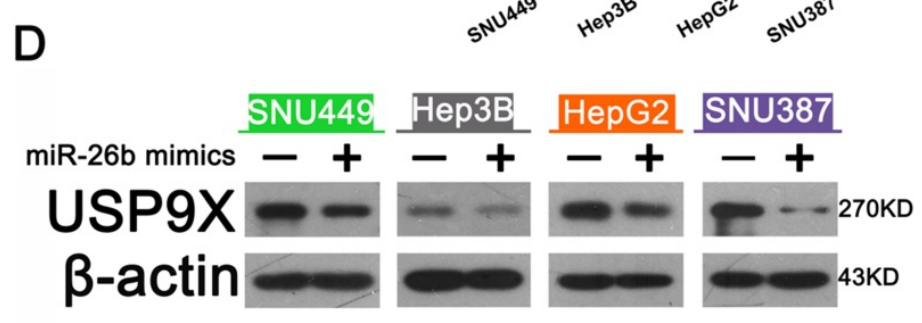

F
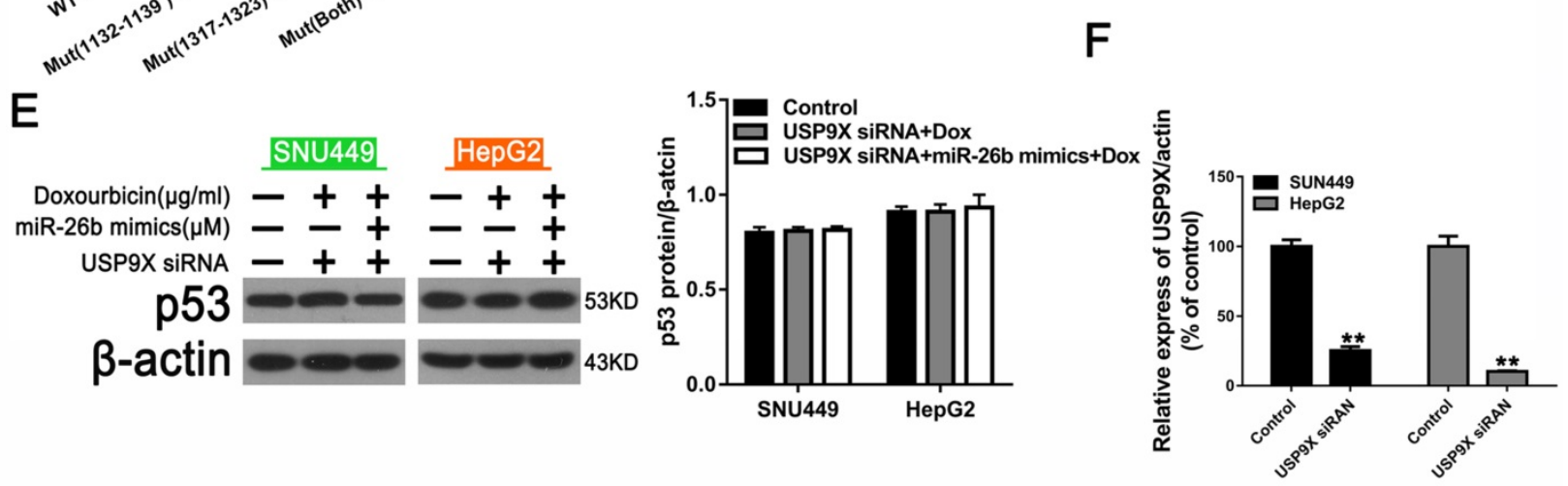

Figure 3. miR-26b enhances HCC cell sensitivity to doxorubicin via USP9X-dependent p53 degradation. A. Western blot indicating the expression of $\mathrm{p} 53$ after treated with doxorubicin, doxorubicin plus miR-26 mimic, or MG 132. **P $<0.01$, ***P $<0.001$. B. Tenovin-1 combined with an miR-26b mimic could enhance doxorubicin sensitivity in HepG 2 cells, except other HCC cells. C. The predicted miR-26b binding site in the USP9X $3^{\prime}$ UTR and dual fluorescence reporter gene experiments verify that miR-26b binds to the promoter region of USP9X. *P $<0.05$, **P $<0.01$, *** $<<0.001$. D. The expression of USP9X was up-regulated following treatment with the miR-26b mimic. $* \mathrm{P}<0.05$, **P $<0.01$. E. P53 protein expression was determined by Western blot. F. The expression of UXP9X was determined by Western Blot. 

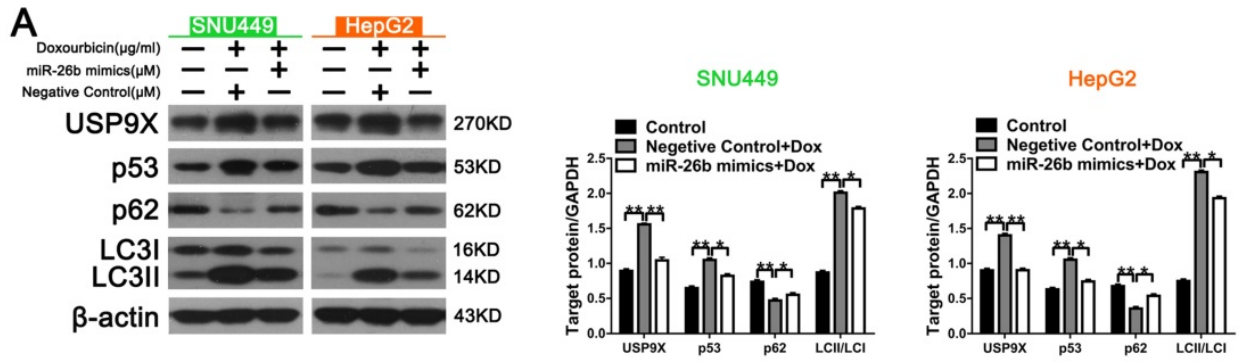

B
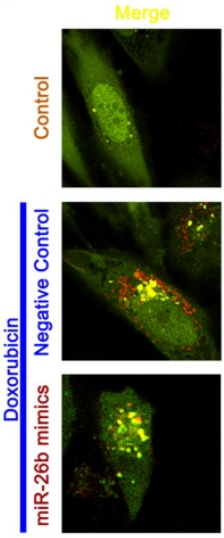

SNU449
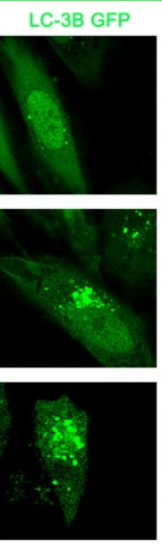
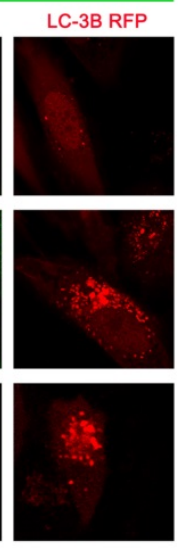

c
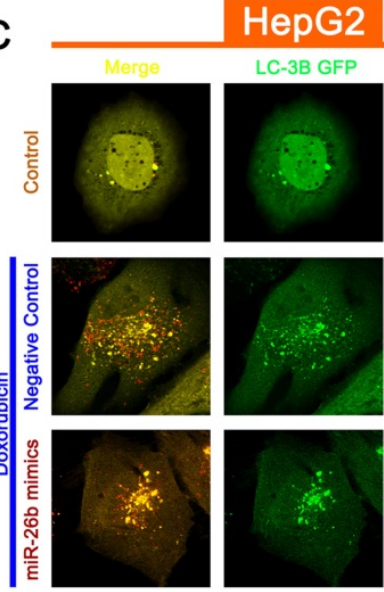

LC-3B RFP
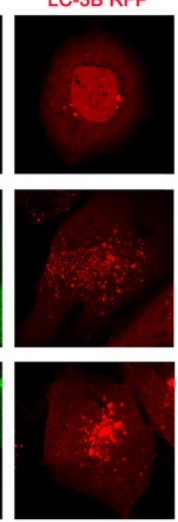

F
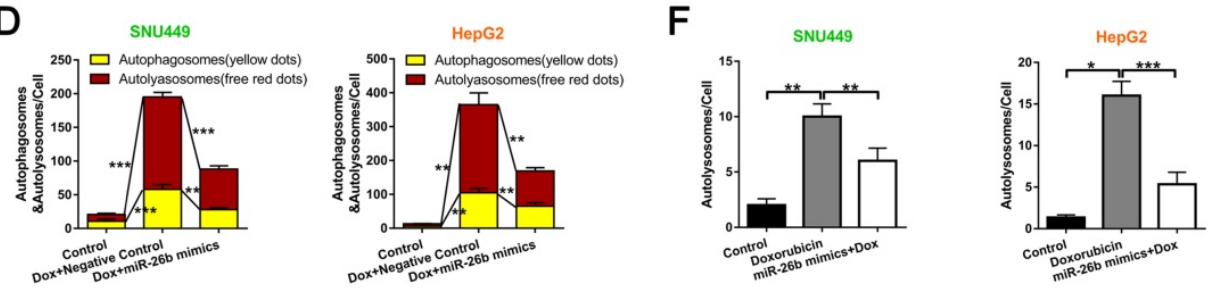

E

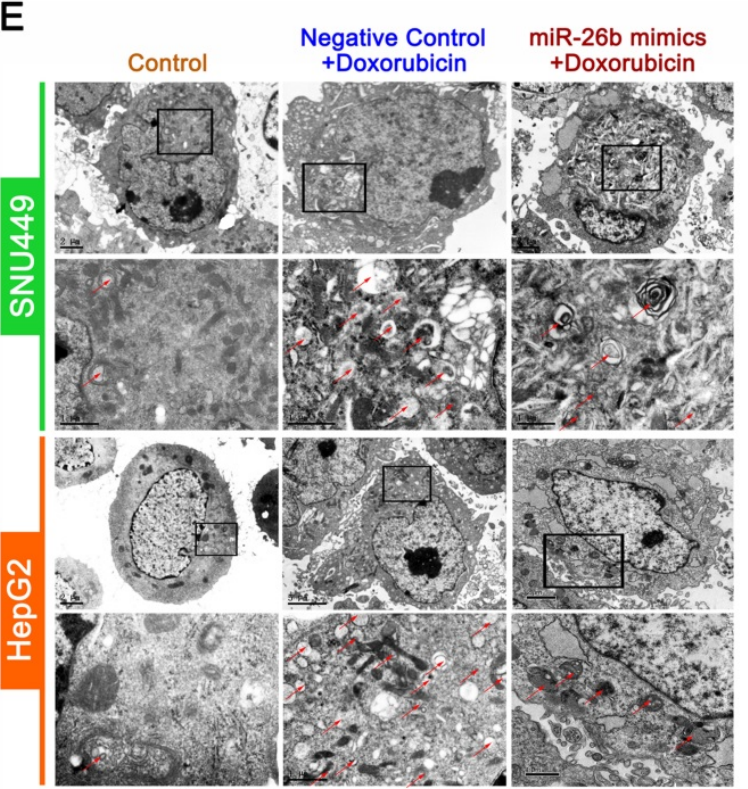

G
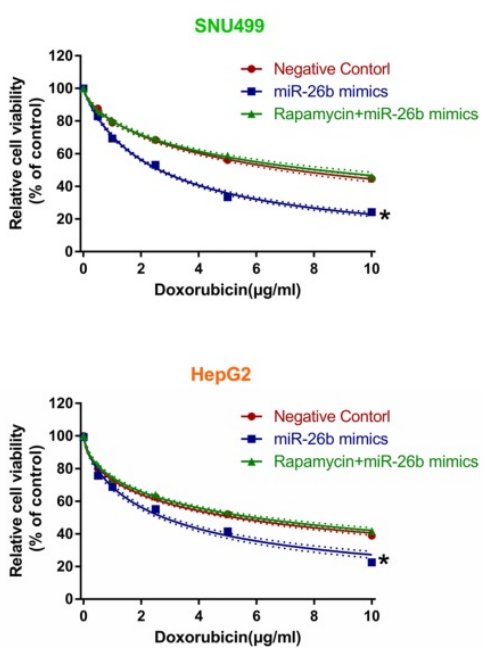

Figure 4. miR-26b enhances doxorubicin sensitivity via autophagy. A. The expression of proteins in HCC cells in different treatment groups detected by Western blot. *P $<0.05$ **P $<0.01$ B-D. Confocal microscopy analysis of LC3 double fluorescent cells *P $<0.05$ * **P $<0.01$; ***P $<0.001$. E-F. Electron microscopy detected autophagic flow in SNU449 and HepG2 cells. **P $<0.01$ G. CCK-8 detected the sensitivity to doxorubicin in HCC cells after treatment with different conditions (transfected with miR-26b mimics, miR-26b mimic interference, and treated with rapamycin). $* \mathrm{P}<0.05$ vs Negative Control. 
A

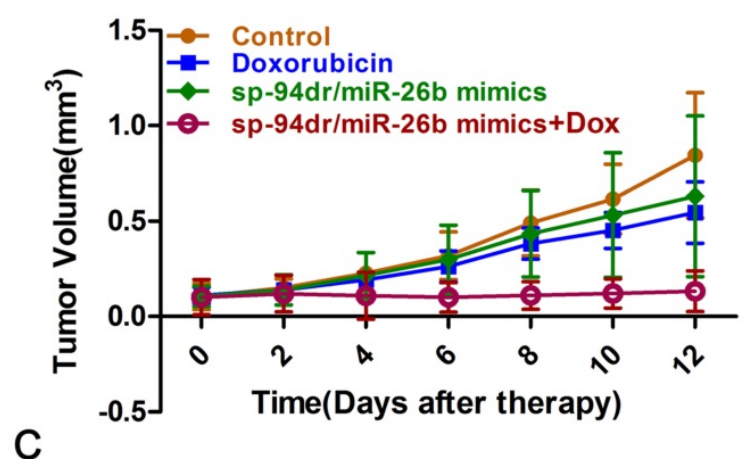

C

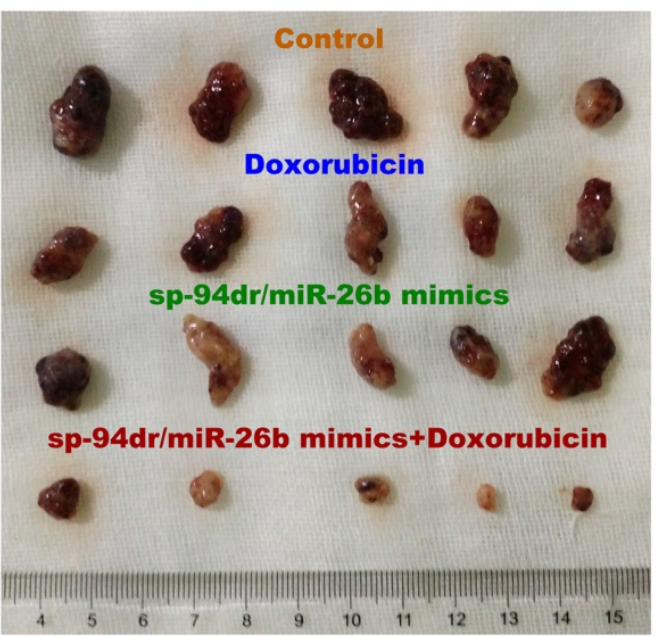

E

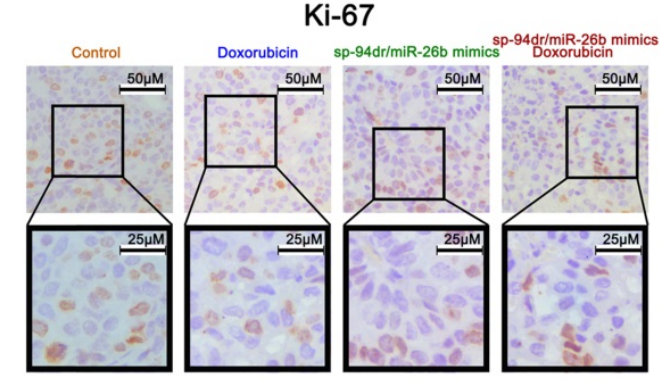

B

\section{body Weight miR-26b}

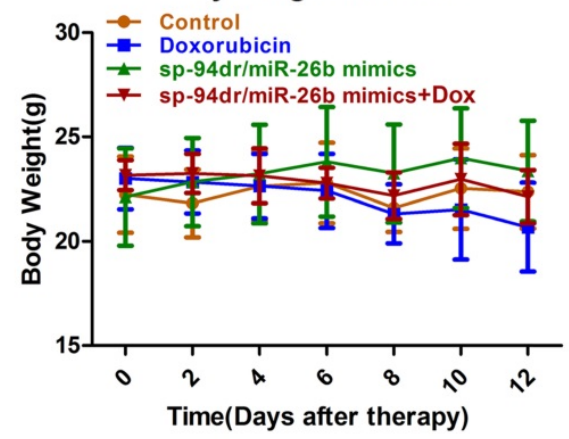

D

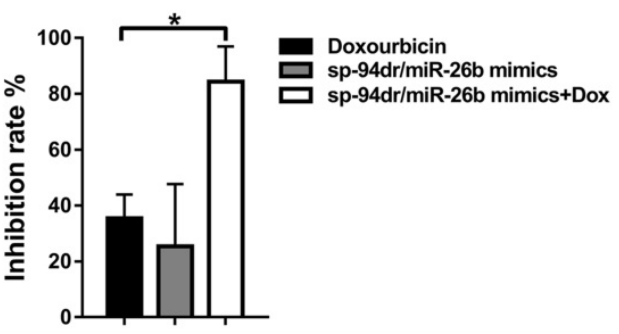

F

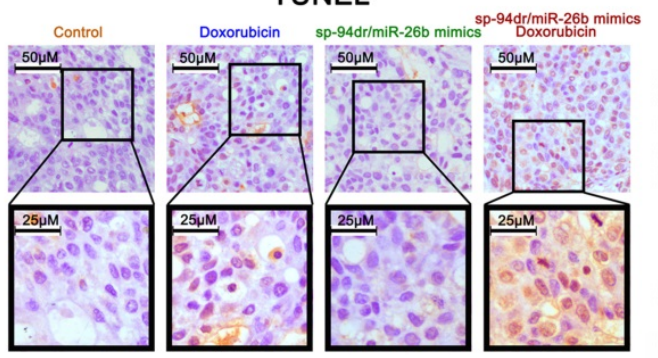

TUNEL

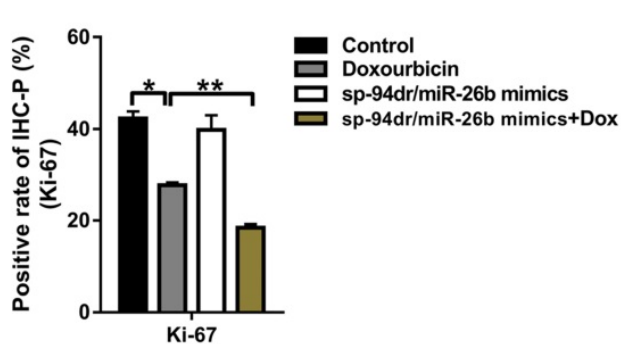


Our previously established chimeric peptidecondensed supramolecular nanoparticles were found to protect miR-26b from degradation [35]. A schematic representation of the binding of miRNA and the polypeptide, SP-94dr, is presented in Supplemental Figure 1 A. The naked miR-26b band was significantly blocked when the SP-94dr/miR-26b ratio was 20:1 (Supplemental Figure 1 B). Agarose gel electrophoresis analysis showed that the RNase stability and miRNA serum stability were significantly increased after binding to SP-94dr (Supplemental Figure $1 \mathrm{C}$ and D). Electron microscopy with the best ratio of nanoparticles (nanoparticles) was used to measure the self-assembled nanoparticles, and an average particle size of $135 \mathrm{~nm} \pm 30 \mathrm{~nm}$ was recorded (Supplemental Figure $1 \mathrm{E}$ and F). Furthermore, treatment with the SP-94dr/ miR-26b mimic nanoparticles plus doxorubicin was found to reduce the level of USP9X and p53 expression (Figure 6A and B). The level of miR-26b and p62 was upregulated following treatment with sp94dr/miR-26b mimic nanoparticles plus doxorubicin, whereas the expression of USP9X and p53 was downregulated compared with the control (Figure 6C). The level of USP9X and p53 protein expression following treated with doxorubicin was increased and p62 expression was decreased compared with the Control; however, the sp94dr/miR-26b mimic nanoparticles could reduce the up-regulation of USP9X and p53 induced by doxorubicin, increase doxorubicin induced the decrease level of p62 (Figure 6D). Taken together, these results suggest that miR-26b increases doxorubicin sensitivity in vivo. Schematic diagram of the regulatory mechanism of the miR-26b/USP9X/ p53 axis in regulating HCC sensitivity to doxorubicin was shown in Figure 7.

\section{Discussion}

Numerous reports have demonstrated that miRNAs play a critical role in developing chemo-resistance based on their functions and targets $[32,33]$. miRNA-26b is encoded at 9p21.3, a vulnerable site in the genome, which has been reported to be missing in many HCC tumors and is downregulated in HCC cells [36]. Moreover, the up-regulation of miR-26b can inhibit cellular proliferation and migration in HCC cells [37] and miR-26b can also suppress tumorigenicity and promote apoptosis in NSCLC cells by regulating Mcl-1 [38]. It has also been shown that miR-26b can improve the sensitivity of colorectal cancer cells to 5 -FU in vitro and enhance the potency of 5-FU on the inhibition of tumor growth in vivo through downregulating P-glycoprotein (Pgp) protein expression [39]. Therefore, determining important candidate miRNAs that regulate HCC chemotherapy resistance may help improve treatment. Our research focused on the role of miR-26b on doxorubicin chemoresistance, demonstrating that miR-26b expression was downregulated in HCC cells. Furthermore, treatment with the miR-26b mimic combined with doxorubicin could enhance the sensitivity to doxorubicin in all HCC cells, except in Hep3B cells. Moreover, our previous study found that WP1130 could enhance the sensitivity to doxorubicin in HCC cells; however, p53 was absent in Hep3B cells [22]. As a tumor suppressor and transcription factor, p53 can regulate different cellular stress responses (e.g., activation of oncogene and DNA damage caused by genotoxic drug) and behaviors (e.g., apoptosis, cell cycle arrest, DNA repair, and cell growth) [40]. The abnormal expression of p53 has been found in almost all types of cancers, and p53 mutations are related to a poor patient prognosis and resistance to chemotherapy [41, 42]. Thus, we examined the expression of p53, and found that p53 expression was almost nonexistent in Hep3B cells. To determine the level of cell viability, p53 was knocked down after transfection with or without an miR-26b mimic plus doxorubicin in HCC cells, revealing that the role of the miR-26b mimic in doxorubicin sensitivity was ablated.

Autophagy is a process of maintaining homeostasis that is conserved in HCC cells following targeted therapy by promoting cell survival, and also induces resistance in a wide-range of cancer cells [29, 43]. Indeed, inhibiting autophagy has been shown to overcome chemoresistance in many tumor cells [27, 44, 45] LC3-I is present in the cytoplasm, while LC3-II is localized inside and outside the membrane of autophagolysosomes [46]. The degradation of LC3-II in the autophagolysosome can be prevented by inhibiting autophagolysosome fusion. The number of LC3-II indicates the number of autophagosomes obtained during the conversion of LC3-I to LC3-II. Autophagy as strictly regulated by autophagy-related molecules and the number of LC3-II or LC3-II/LC3-I can reflect autophagy activity [47, 48]. p62 is one of the most well-characterized autophagy receptors, and degradation via autophagy was found to be selected by linking cargo with autophagy mechanism [49]. It has been reported that the up-regulation of miR-26b may enhance the sensitivity to doxorubicin and promote apoptosis via inhibiting autophagy in HCC cells [29]. Furthermore, the main regulators of autophagy include the PI3K-Akt-mTOR pathwayassociated molecules, RAS and p53 [25]. Several studies have shown that nuclear p53 stimulates cellular autophagy via the transactivation of multiple 
target genes, whereas cytoplasmic p53 inhibits autophagy in a transcription-independent manner. Therefore, the subcellular localization of p53 may determine the outcome of autophagy [50]. Here, we investigated the role of autophagy in miR-26bmediated regulation of doxorubicin resistance in HCC. Almost immediately, we examined LC3, double fluorescence and autophagic flow following transfection with an miR-26b mimic, and miR-26b mimic combined with doxorubicin. Interestingly, we found that the miR-26b mimic reduced the increased rate of LC3-II/LC3-I induced by doxorubicin and increased p62 expression, indicating that miR-26b upregulation suppressed autophagy. We subsequently found that following treatment with rapamycin combined with an miR-26b mimic, the increased doxorubicin sensitivity mediated by miR-26b had disappeared, suggesting that autophagy may be related to drug resistance in HCC cells.

A

USP9X
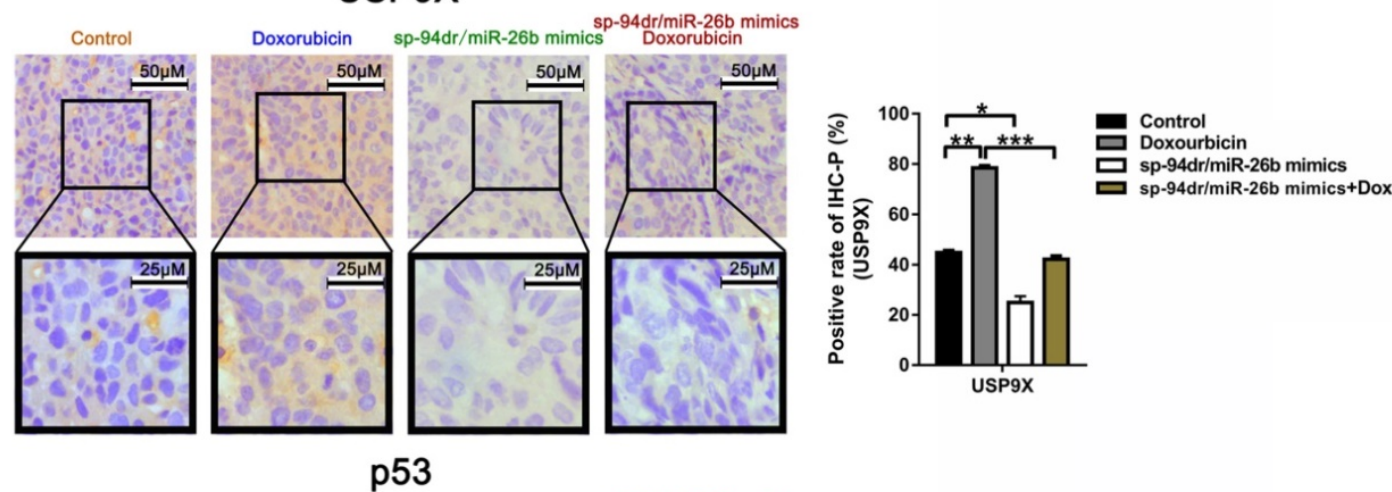

B
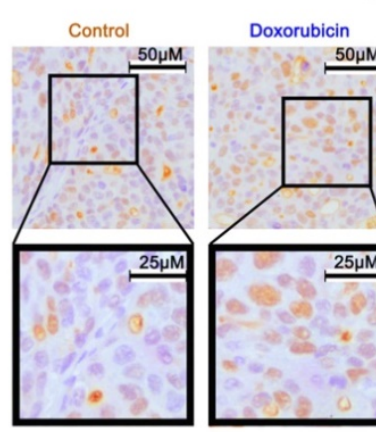

p53

C
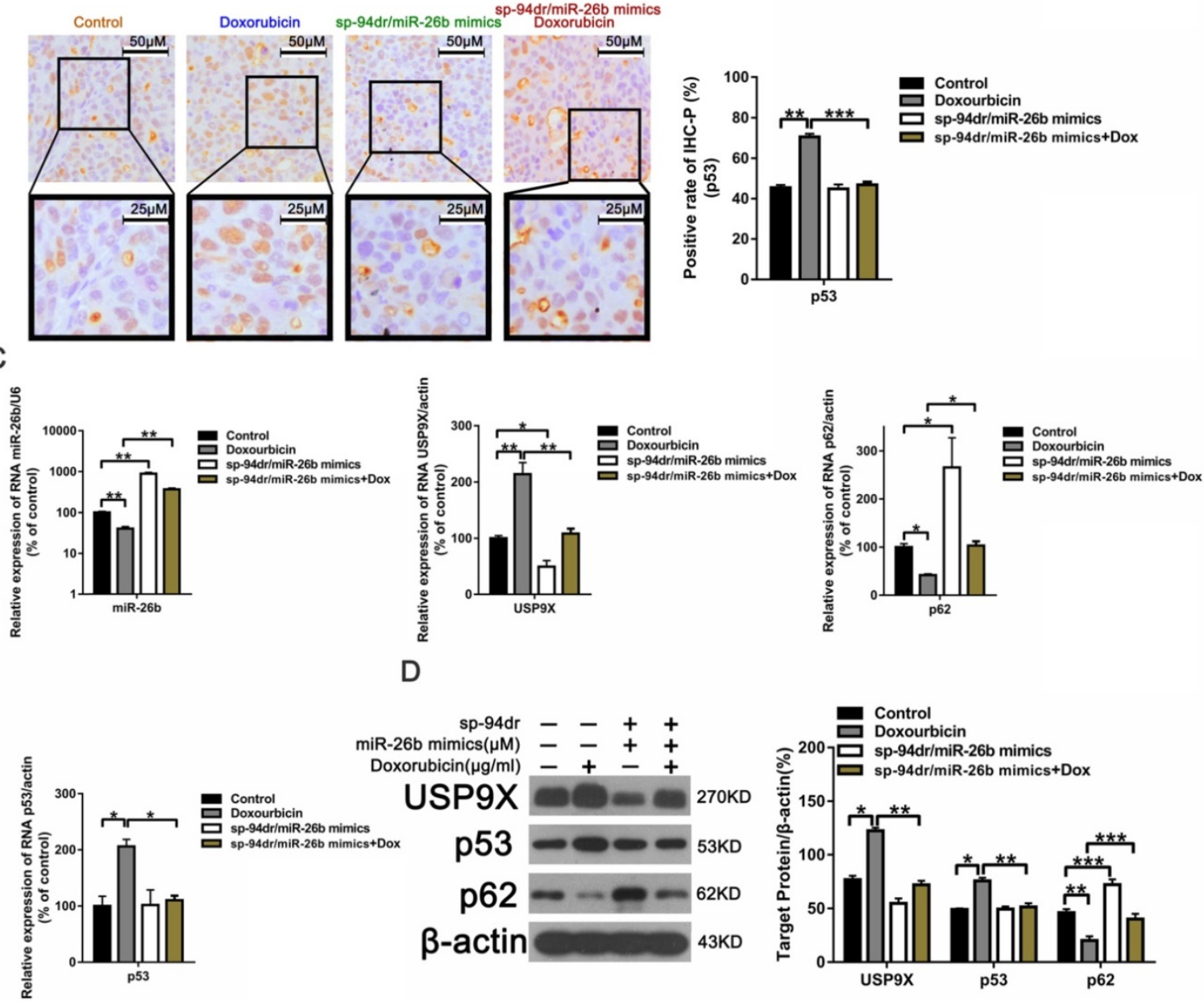

D
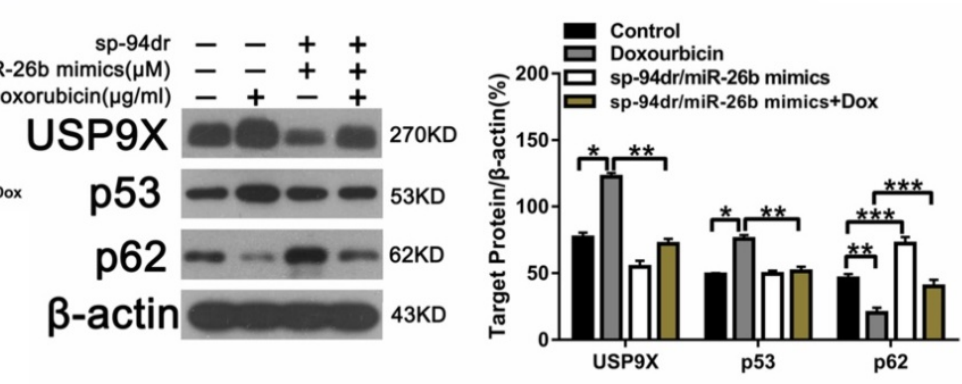

Figure 6. Treatment with Sp-94dr/miR-26b mimic combined with doxorubicin down-regulates p53 and USP9X expression in vivo. A and B. Immunohistochemistry-positive (IHC-P) cells were examined for the expression of USP9X and $\mathrm{P} 53$. *P $<0.05 ; * * \mathrm{P}<0.01 ; * * * \mathrm{P}<0.001$. C. QRT-PCR analysis the expression of $\mathrm{miR}-26 \mathrm{~b}$, USP9X, and $\mathrm{p} 53$ in control, doxorubicin, sp-94dr/miR-26b mimic, and doxorubicin plus with sp-94dr/miR-26b mimic-treated cells. *P < 0.05 ; $* * \mathrm{P}<0.01$; $* * * \mathrm{P}<0.001$. D. USP9X, $\mathrm{P} 53$, and $\mathrm{p} 62$ protein expression were evaluated using a Western blot. $* \mathrm{P}<0.05 ; * * \mathrm{P}<0.01$; ***P $<0.001$. 
Protein ubiquitination is regulated by various aspects of cellular physiology, including cell signaling and protein degradation, and it is also a reversible, post-translational modification [51]. Moreover, DUBs can cleave ubiquitin from the substrate [52]. In the human genome, approximately 100 encoded DUBs have been found, of which USP9X/FAM has been implicated in multiple physiological pathways [53]. Accumulating evidence has revealed that USP9X is significantly up-regulated in human cancers, including pancreatic, breast, HCC, and lung cancers [54-57]. Moreover, USP9X overexpression was found to be closely associated to apoptosis, metastasis, cellular proliferation, and chemotherapy resistance $[54,58]$. In this study, USP9X interference was shown to enhance HCC cell sensitivity to doxorubicin; however, the role of the miR-26b mimic disappeared. p53 has been determined to be the most common variant gene in cancers, and its regulation and stabilization of normal functions is essential. In addition, the ubiquitin-proteasome pathway (UPP) regulates p53 stability and is modulated by DUBs that can eliminate ubiquitin from p53. The ubiquitination of p53 can be reversed by DUBs, a group of enzymes that control the extent of p53 ubiquitination through deubiquitinating p53. Both ubiquitination or deubiquitylation regulate p53 inside the cells [59-61]. MG132 acts as a proteasome inhibitor, further verifying that p53 downregulation was achieved by proteasome-dependent degradation via p53 ubiquitination. To further explore whether miR-26b mediates its synergistic cytotoxicity through p53 degradation, we treated cells with doxorubicin alone, or in combination with an miR-26b mimic or MG132 with doxorubicin and an miR-26b mimic. The results showed that MG132 reversed the inhibitory effect of miR-26b on p53 expression in the HCC cells following doxorubicin treatment. However, no significant differences in p53 expression were observed after treatment with the miR-26b mimic, suggesting that miR-26b promoted p53 degradation via USP9X. It has been reported that Tenovin-1 can protect p53 from degradation via $\mathrm{mdm} 2$, has little effect on p53 synthesis, and has also been identified as a p53 activator [22, 62]. In addition, this effect was only found in wild type p53 but not in p53 mutant cells [63]. Our study showed that Tenovin- 1 protects p53 from ubiquitination-mediated degradation only in wild type p53 HepG2 cells. Tenovin-1 pretreatment enhances HCC cell resistance to doxorubicin following co-treatment with an miR-26b mimic, indicating that the USP9X/p53/ubiquitination/ degradation pathway is important for miR-26bmediated chemotherapy sensitivity. Furthermore, we established chimeric peptide-condensed supramolecular nanoparticles to protect miR-26b from degradation. Later, in vivo treatment with sp94dr/miR-26b mimic nanoparticles plus dox was associated with inhibitory effects on tumorigenesis. Furthermore, miR-26b was up-regulated, whereas USP9X and p53 expression were down-regulated, suggesting that miR-26b increases doxorubicin sensitivity via inhibiting USP9X and p53 in vivo.

\section{Conclusion}

In conclusion, this study revealed that miR-26b regulation was a mechanism involved in the sensitivity of HCC cell lines to doxorubicin directly through USP9X-dependent degradation of p53 and regulation of autophagy (Figure 7). To our knowledge, this is the first report of the regulation of USP9X by miR-26b and its role in the chemotherapy response to doxorubicin. Furthermore, miR-26b may represent a potential gene-targeting approach for HCC treatment in the future.

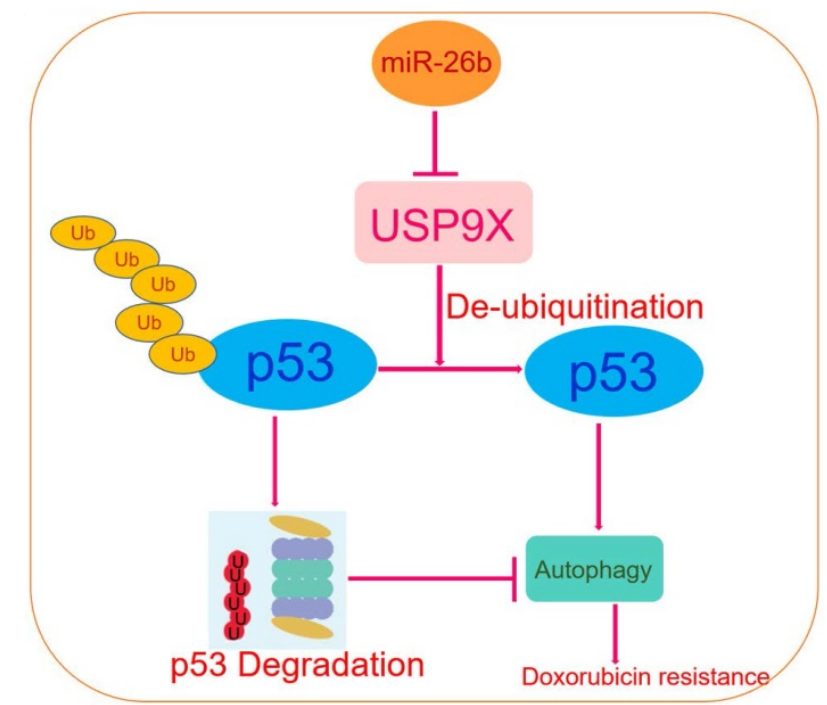

Figure 7. Schematic diagram of the regulatory mechanism of the miR-26b/USP9X/p53 axis in regulating HCC sensitivity to doxorubicin.

\section{Supplementary Material}

Supplementary figure $\mathrm{S} 1$.

http://www.ijbs.com/v17p0781s1.pdf

\section{Acknowledgements}

This work was supported by the National Natural Science Foundation of China (81830089, 81901690, 81802383 and 81701630), Zhejiang Provincial Innovation Center for the Study of Pancreatic Disease, Zhejiang Clinical Research Center of Hepatobiliary and Pancreatic Diseases, Hangzhou, China, Zhejiang Provincial Medical and Health Research Project (2018KY126), Projects of Lishui Key Research and Development Plan in Zhejiang Province 


\section{(2017ZDYF12).}

\section{Ethics approval and consent to participate}

All animal experiments were approved by the Medical Ethics Committee of Zhejiang University and conformed to the National Institutes of Health Guide for Care and Use of Laboratory Animals (NIH Publications, No. 8023, revised 1978).

\section{Competing Interests}

The authors have declared that no competing interest exists.

\section{References}

1. Bruix J, Gores GJ, Mazzaferro V. Hepatocellular carcinoma: clinical frontiers and perspectives. Gut. 2014; 63: 844-55.

2. Gao J, Inagaki $\mathrm{Y}$, Song $\mathrm{P}, \mathrm{Qu} \mathrm{X}$, Kokudo $\mathrm{N}$, Tang $\mathrm{W}$. Targeting c-Met as a promising strategy for the treatment of hepatocellular carcinoma. Pharmacol Res. 2012; 65: 23-30.

3. Ma J, Zeng S, Zhang Y, Deng G, Qu Y, Guo C, et al. BMP4 promotes oxaliplatin resistance by an induction of epithelial-mesenchymal transition via MEK1/ERK/ELK1 signaling in hepatocellular carcinoma. Cancer Lett. 2017; 411: 117-29.

4. Bartel DP. MicroRNAs: genomics, biogenesis, mechanism, and function. Cell. 2004; 116: 281-97.

5. Bartel DP. MicroRNAs: target recognition and regulatory functions. Cell. 2009; 136: 215-33.

6. Morishita A, Masaki T. miRNA in hepatocellular carcinoma. Hepatol Res. 2015; 45: 128-41.

7. Climent J, Dimitrow P, Fridlyand J, Palacios J, Siebert R, Albertson DG, et al. Deletion of chromosome $11 \mathrm{q}$ predicts response to anthracycline-based chemotherapy in early breast cancer. Cancer Res. 2007; 67: 818-26.

8. Garofalo M, Quintavalle C, Di Leva G, Zanca C, Romano G, Taccioli C, et al. MicroRNA signatures of TRAIL resistance in human non-small cell lung cancer. Oncogene. 2008; 27: 3845-55.

9. Wagenaar TR, Zabludoff S, Ahn SM, Allerson C, Arlt H, Baffa R, et al. Anti-miR-21 Suppresses Hepatocellular Carcinoma Growth via Broad Transcriptional Network Deregulation. Mol Cancer Res. 2015; 13: 1009-21.

10. Simerzin A, Zorde-Khvalevsky E, Rivkin M, Adar R, Zucman-Rossi J, Couchy $\mathrm{G}$, et al. The liver-specific microRNA-122*, the complementary strand of microRNA-122, acts as a tumor suppressor by modulating the p53/mouse double minute 2 homolog circuitry. Hepatology. 2016; 64: 1623-36.

11. Xia M, Duan ML, Tong JH, Xu JG. MiR-26b suppresses tumor cell proliferation, migration and invasion by directly targeting COX-2 in lung cancer. Eur Rev Med Pharmacol Sci. 2015; 19: 4728-37.

12. Li H, Sun $\mathrm{Q}, \mathrm{Han} B, \mathrm{Yu}$ X, Hu B, Hu S. MiR-26b inhibits hepatocellular carcinoma cell proliferation, migration, and invasion by targeting EphA2. Int $J$ Clin Exp Pathol. 2015; 8: 4782-90.

13. Li M, Long C, Yang G, Luo Y, Du H. MiR-26b inhibits melanoma cell proliferation and enhances apoptosis by suppressing TRAF5-mediated MAPK activation. Biochem Biophys Res Commun. 2016; 471: 361-7.

14. Lin J, Zhang L, Huang $H$, Huang $Y$, Huang $L$, Wang J, et al. MiR-26b/KPNA2 axis inhibits epithelial ovarian carcinoma proliferation and metastasis through downregulating OCT4. Oncotarget. 2015; 6: 23793-806.

15. Zhang C, Tong J, Huang G. Nicotinamide phosphoribosyl transferase (Nampt) is a target of microRNA-26b in colorectal cancer cells. PLoS One. 2013; 8: e69963.

16. Sun J, Yan P, Chen Y, Chen Y, Yang J, Xu G, et al. MicroRNA-26b inhibits cell proliferation and cytokine secretion in human RASF cells via the Wnt/GSK-3beta/beta-catenin pathway. Diagn Pathol. 2015; 10: 72

17. Kato M, Goto Y, Matsushita R, Kurozumi A, Fukumoto I, Nishikawa R, et al. MicroRNA-26a/b directly regulate La-related protein 1 and inhibit cancer cell invasion in prostate cancer. Int J Oncol. 2015; 47: 710-8.

18. Xu G, Ji C, Song G, Shi C, Shen Y, Chen L, et al. Obesity-associated microRNA-26b regulates the proliferation of human preadipocytes via arrest of the G1/S transition. Mol Med Rep. 2015; 12: 3648-54.

19. Kapuria V, Peterson LF, Fang D, Bornmann WG, Talpaz M, Donato NJ. Deubiquitinase inhibition by small-molecule WP1130 triggers aggresome formation and tumor cell apoptosis. Cancer Res. 2010; 70: 9265-76.

20. Kerscher O, Felberbaum R, Hochstrasser M. Modification of proteins by ubiquitin and ubiquitin-like proteins. Annu Rev Cell Dev Biol. 2006; 22: $159-80$.

21. Schwickart M, Huang X, Lill JR, Liu J, Ferrando R, French DM, et al. Deubiquitinase USP9X stabilizes MCL1 and promotes tumour cell survival. Nature. 2010; 463: 103-7.
22. Liu H, Chen W, Liang C, Chen BW, Zhi X, Zhang S, et al. WP1130 increases doxorubicin sensitivity in hepatocellular carcinoma cells through usp9x-dependent p53 degradation. Cancer Lett. 2015; 361: 218-25.

23. Wang X, Bao Y, Dong Z, Chen Q, Guo H, Ziang C, et al. WP1130 attenuates cisplatin resistance by decreasing P53 expression in non-small cell lung carcinomas. Oncotarget. 2017; 8: 49033-43.

24. Peddaboina C, Jupiter D, Fletcher S, Yap JL, Rai A, Tobin RP, et al. The downregulation of Mcl-1 via USP9X inhibition sensitizes solid tumors to Bcl-xl inhibition. BMC Cancer. 2012; 12: 541.

25. Chen L, Zhou Y, Sun Q, Zhou J, Pan H, Sui X. Regulation of Autophagy by MiRNAs and Their Emerging Roles in Tumorigenesis and Cancer Treatment. Int Rev Cell Mol Biol. 2017; 334: 1-26.

26. Xu X, Tao Y, Shan L, Chen R, Jiang H, Qian Z, et al. The Role of MicroRNAs in Hepatocellular Carcinoma. J Cancer. 2018; 9: 3557-69.

27. Huang H, Tang J, Zhang L, Bu Y, Zhang X. miR-874 regulates multiple-drug resistance in gastric cancer by targeting ATG16L1. Int J Oncol. 2018; 53: 2769-79.

28. Hua L, Zhu G, Wei J. MicroRNA-1 overexpression increases chemosensitivity of non-small cell lung cancer cells by inhibiting autophagy related 3-mediated autophagy. Cell Biol Int. 2018; 42: 1240-9.

29. Jin $\mathrm{F}$, Wang $\mathrm{Y}$, Li M, Zhu $\mathrm{Y}$, Liang $\mathrm{H}$, Wang $\mathrm{C}$, et al. MiR-26 enhances chemosensitivity and promotes apoptosis of hepatocellular carcinoma cells through inhibiting autophagy. Cell Death Dis. 2017; 8: e2540.

30. Du X, Liu B, Luan X, Cui Q, Li L. miR-30 decreases multidrug resistance in human gastric cancer cells by modulating cell autophagy. Exp Ther Med. 2018; 15: 599-605.

31. Tu C, Chen W, Wang S, Tan W, Guo J, Shao C, et al. MicroRNA-383 inhibits doxorubicin resistance in hepatocellular carcinoma by targeting eukaryotic translation initiation factor 5A2. J Cell Mol Med. 2019; 23: 7190-9.

32. Qu YM, Sun X, Yan XL, Jin H, Guo ZN, Yang Y. Identification of microRNAs and messenger RNAs involved in human umbilical cord mesenchymal stem cell treatment of ischemic cerebral infarction using integrated bioinformatics analysis. Neural Regen Res. 2019; 14: 1610-6.

33. Oh SH, Choi YB, Kim JH, Weihl CC, Ju JS. Quantification of autophagy flux using LC3 ELISA. Anal Biochem. 2017; 530: 57-67.

34. Pugsley HR. Assessing Autophagic Flux by Measuring LC3, p62, and LAMP1 Co-localization Using Multispectral Imaging Flow Cytometry. J Vis Exp. 2017.

35. Chen W, Zhou Y, Zhi X, Ma T, Liu H, Chen BW, et al. Delivery of miR-212 by chimeric peptide-condensed supramolecular nanoparticles enhances the sensitivity of pancreatic ductal adenocarcinoma to doxorubicin. Biomaterials. 2019; 192: 590-600

36. Zhu Y, Lu Y, Zhang Q, Liu JJ, Li TJ, Yang JR, et al. MicroRNA-26a/b and their host genes cooperate to inhibit the G1/S transition by activating the pRb protein. Nucleic Acids Res. 2012; 40: 4615-25.

37. Lin CZ, Ou RW, Hu YH. Lentiviral-mediated microRNA-26b up-regulation inhibits proliferation and migration of hepatocellular carcinoma cells. Kaohsiung J Med Sci. 2018; 34: 547-55.

38. $\mathrm{Yu}$ JG, Ji CH, Shi $\mathrm{MH}$. MicroRNA-26b suppresses tumorigenicity and promotes apoptosis in small cell lung cancer cells by targeting myeloid cell leukemia 1 protein. Kaohsiung J Med Sci. 2018; 34: 593-605.

39. Wang B, Lu FY, Shi RH, Feng YD, Zhao XD, Lu ZP, et al. MiR-26b regulates 5-FU-resistance in human colorectal cancer via down-regulation of Pgp. Am J Cancer Res. 2018; 8: 2518-27.

40. Marte B. Cancer: super p53. Nature. 2002; 420: 279 .

41. Soussi T, Beroud C. Assessing TP53 status in human tumours to evaluate clinical outcome. Nat Rev Cancer. 2001; 1: 233-40.

42. Shetzer Y, Solomon H, Koifman G, Molchadsky A, Horesh S, Rotter V. The paradigm of mutant p53-expressing cancer stem cells and drug resistance. Carcinogenesis. 2014: 35: 1196-208.

43. Chen R, Wang H, Liang B, Liu G, Tang M, Jia R, et al. Downregulation of ASPP2 improves hepatocellular carcinoma cells survival via promoting BECN1-dependent autophagy initiation. Cell Death Dis. 2016; 7: e2512.

44. Liu J, Xing Y, Rong L. miR-181 regulates cisplatin-resistant non-small cell lung cancer via downregulation of autophagy through the PTEN/PI3K/AKT pathway. Oncol Rep. 2018; 39: 1631-9.

45. Zou J, Liu L, Wang $\mathrm{Q}$, Yin F, Yang Z, Zhang W, et al. Downregulation of miR-429 contributes to the development of drug resistance in epithelial ovarian cancer by targeting ZEB1. Am J Transl Res. 2017; 9: 1357-68.

46. Khandelwal VK, Mitrofan LM, Hyttinen JM, Chaudhari KR, Buccione R, Kaarniranta K, et al. Oxidative stress plays an important role in zoledronic acid-induced autophagy. Physiol Res. 2014; 63 Suppl 4: S601-12.

47. Tanida I, Ueno T, Kominami E. LC3 and Autophagy. Methods Mol Biol. 2008; 445: 77-88

48. Wang J, Liu Y, Li XH, Zeng XC, Li J, Zhou J, et al. Curcumin protects neuronal cells against status-epilepticus-induced hippocampal damage through induction of autophagy and inhibition of necroptosis. Can J Physiol Pharmacol. 2017; 95: 501-9.

49. Mijaljica D, Nazarko TY, Brumell JH, Huang WP, Komatsu M, Prescott M, et al. Receptor protein complexes are in control of autophagy. Autophagy. 2012; 8: 1701-5.

50. Schmukler E, Kloog Y, Pinkas-Kramarski R. Ras and autophagy in cancer development and therapy. Oncotarget. 2014; 5: 577-86.

51. Kudo-Saito C, Shirako H, Takeuchi T, Kawakami Y. Cancer metastasis is accelerated through immunosuppression during Snail-induced EMT of cancer cells. Cancer Cell. 2009; 15: 195-206. 
52. Imamura T, Oshima $Y$, Hikita A. Regulation of TGF-beta family signalling by ubiquitination and deubiquitination. J Biochem. 2013; 154: 481-9.

53. Yang CC, Wolf DA. Inflamed snail speeds metastasis. Cancer Cell. 2009; 15: 355-7.

54. Oosterkamp HM, Hijmans EM, Brummelkamp TR, Canisius S, Wessels LF, Zwart W, et al. USP9X downregulation renders breast cancer cells resistant to tamoxifen. Cancer Res. 2014; 74: 3810-20.

55. Liu L, Yao D, Zhang P, Ding W, Zhang X, Zhang C, et al. Deubiquitinase USP9X promotes cell migration, invasion and inhibits apoptosis of human pancreatic cancer. Oncol Rep. 2017; 38: 3531-7.

56. Chen H, Yang F, Li X, Gong ZJ, Wang LW. Long noncoding RNA LNC473 inhibits the ubiquitination of survivin via association with USP9X and enhances cell proliferation and invasion in hepatocellular carcinoma cells. Biochem Biophys Res Commun. 2018; 499: 702-10.

57. Peng J, Hu Q, Liu WP, Liu SL, Wang H. [Expression of USP9X in Non-small Cell Lung Cancer and Its Clinical Significance]. Sichuan Da Xue Xue Bao Yi Xue Ban. 2015; 46: 413-6.

58. Perez-Mancera PA, Rust AG, van der Weyden L, Kristiansen G, Li A, Sarver $\mathrm{AL}$, et al. The deubiquitinase USP9X suppresses pancreatic ductal adenocarcinoma. Nature. 2012; 486: 266-70.

59. Brooks CL, Gu W. p53 ubiquitination: Mdm2 and beyond. Mol Cell. 2006; 21: 307-15.

60. Kwon SK, Saindane M, Baek KH. p53 stability is regulated by diverse deubiquitinating enzymes. Biochim Biophys Acta Rev Cancer. 2017; 1868: 404-11.

61. Dar A, Shibata E, Dutta A. Deubiquitination of Tip60 by USP7 determines the activity of the p53-dependent apoptotic pathway. Mol Cell Biol. 2013; 33: 3309-20.

62. Raza S, Ohm JE, Dhasarathy A, Schommer J, Roche C, Hammer KD, et al. The cholesterol metabolite 27-hydroxycholesterol regulates p53 activity and increases cell proliferation via MDM2 in breast cancer cells. Mol Cell Biochem. 2015; 410: 187-95.

63. Sonnemann J, Marx C, Becker S, Wittig S, Palani CD, Kramer OH, et al. p53-dependent and p53-independent anticancer effects of different histone deacetylase inhibitors. Br J Cancer. 2014; 110: 656-67. 